\title{
De la Francophonie "centripète" à une Francophonie périphérique
}

\author{
Virginie Marie \\ Université de Turku \\ Laboratoire CERCI (Université de Nantes)
}

\section{Contexte néo-colonialiste}

L'existence même du concept de la Francophonie au sens culturel et politique du terme est relativement récente puisque comme nous le savons, elle provient directement du processus de décolonisation remontant à la fin des années 1950 et au début des années 1960. Cependant, les termes de «francophone » et de «francophonie » paraissent beaucoup plus anciens : en effet leurs premières occurrences datent de la fin du XIXe siècle et les dictionnaires estiment à 1880 la date de leur entrée dans la langue. Selon les principaux auteurs qui se sont intéressés aux origines de ces termes, plus particulièrement Xavier Deniau, Claude Hagège, Michel Tétu, attestent que le premier auteur à les avoir utilisés est le géographe Onésime Reclus, qui avait orienté ses travaux essentiellement vers l'étude de la répartition des langues dans le monde. La Francophonie naît donc de l'idée d'une classification des habitants de la planète en tenant compte d'une variable déterminante, celle de la langue parlée au sein de la cellule familiale ou dans un cercle plus élargi aux relations sociales. Ainsi, Onésime Reclus reconnu comme le premier auteur à avoir utilisé les termes «francophone » et "francophonie » est souvent présenté comme porte-parole d'un dialogue des cultures. L'étude approfondie de son ouvrage France, Algérie et colonies (paru en 1886), consacrée à la théorisation de la colonisation républicaine à la fin du XIXe siècle, met en avant l'idée d'un nationalisme convaincu, assuré de préserver la langue française et la France d'un éventuel déclin démographique (Onésime 1886). La Francophonie désigne pour Onésime Reclus l'ensemble des populations parlant le français. L'auteur s'intéresse à l'universalité de la langue française, à l'Algérie, aux colonies et anciennes colonies de France. Onésime Reclus entend ainsi par francophonie "tous ceux qui sont ou semblent être destinés à rester ou à devenir participants de notre langue " (Onésime 1886). Pour reprendre les propos de Deniau : " Le sens premier et immédiat du terme était né : la francophonie recouvrait à la fois une idée linguistique et une relation géographique (ensemble où l'on parle français). O. Reclus entrevoyait une francophonie, symbole et résumé de la solidarité humaine, du partage de la culture et de l'échange" (Deniau 10). L'approche de l'auteur perpétuait un discours épilinguistique d'expansion du francien hors de l'île de France. Elle confirmait également cette croyance populaire qui faisait de la langue française la propriété de la France. Il faut rappeler que cette conception traditionnaliste de la Francophonie est héritée directement du siècle des Lumières 
(Descartes, Voltaire) qui avait une exigence d'universalité dotée d'une volonté d'unité et d'unification hégémonique.

Cependant, le mot et l'idée de la Francophonie vont s'estomper de la conscience collective et de tous les écrits. D'autres mots le remplacent comme "francité", "francophilie", "francitude", "communauté francophone". Il faut attendre 1962, date de l'indépendance algérienne pour redécouvrir ce terme qui sera employé par Léopold Sédar Senghor ${ }^{1}$ (président de la République du Sénégal) dans un numéro spécial de la revue Esprit intitulé "Le français dans le monde" : "Cet humanisme intégral, qui se tisse autour de la terre ; cette symbiose des "énergies dormantes" de tous les continents, de toutes les races, qui se réveillent à leur chaleur complémentaire [...] " (Deniau 11). L'auteur-poète était ainsi convaincu que la "civilisation de l'universel", faisant référence ici à la francophonie dans son ensemble le plus large, devait s'étendre non seulement sur un plan culturel mais également politique. Sous sa plume, le terme "francophone" va progressivement acquérir un caractère linguistique, géographique, culturel et économique, auquel s'ajoute une dimension politique. De ce fait, le projet de la francophonie, englobant ici l'ensemble de gouvernements participant à la communauté francophone, prend de l'importance et s'organise afin de permettre aux différents pays d'expression française de participer à une même "communauté d'espérance et de destin". Mais l'évolution du concept francophone ne semble pas s'arrêter ici car au-delà de la valeur philosophique et morale attribuée à la langue française, certains ont fait de l'usage du français un principe d'action politique : "Je crois à l'avenir de la langue française parce que je crois à l'avenir de la France" proclame Farandjis², Secrétaire Général du Haut Conseil de la Francophonie, dans une profession de foi idéologique. Léopold Sédar Senghor instaure l'utilisation obligée du français en principe de morale et d'action : "La francité n'est certes pas une nouvelle manière de penser; elle est une nouvelle manière de concevoir et d'agir, puisque nous voilà au seuil de la société industrielle : au seuil de l'action logique, de l'action dialectique, surtout de l'action efficace ${ }^{3}$."

Ainsi, les situations de francophonie évoquées ci-dessus doivent être prises en compte afin d'en dégager des sens divers qui nous permettront de classer les définitions de ces différents concepts. Dans notre cas, aborder les situations de l'espace francophone répond certes à un besoin scientifique mais aussi à des besoins d'informations. En répertoriant les différentes

\footnotetext{
${ }^{1}$ Contradictoirement, c'est hors de France et parmi les dirigeants des pays colonisés qu'a émergé l'idée de francophonie, en vue de constituer et d'organiser un ensemble autour des peuples ayant la langue française en partage, à l'image de personnalités illustres comme Léopold Sédar Senghor (Sénégal), Habib Bourguiba (Tunisie), Hamani Diori (Niger) et du Prince Norodom Sihanouk (Cambodge).

2 Textes et propos sur la francophonie, préface de Léopold Sédar Senghor, Paris, Editions Richelieu-Senghor, 1986.

${ }^{3}$ Discours du 17 mars 1967 prononcé à la tribune de l'Assemblée Internationale des Parlementaires de Langue Française.
} 
analyses typologiques des situations linguistiques chez les auteurs cités ci-dessus, cela nous amène à clarifier les différents concepts attribués à la Francophonie en tenant compte des diverses classifications qui lui sont associées. De nos jours, et ce depuis l'institutionnalisation du fait francophone, quatre sens peuvent être attribués au mot «Francophonie », selon la classification faite par Deniau :

1) "un sens linguistique : le substantif tire son origine de l'adjectif « francophone » signifiant « qui parle la langue française » ou «personne parlant le français ». Au sein de ce contexte, la Francophonie se définit comme le "fait d'être francophone », 2) un sens géographique : l'ensemble des peuples, des hommes dont la langue (maternelle, officielle, courante ou administrative) est le français, 3) un sens spirituel et mystique : le sentiment d'appartenir à une même communauté. Une solidarité qui naît du partage de valeurs communes aux divers individus et communautés francophones, 4) un sens institutionnel : l'ensemble des organisations publiques et privées s'attache à défendre et valoriser l'espace francophone " (Deniau 15-24).

Dans un souci de simplification et de synthétisation, nous résumerons cette classification faite par Deniau, en ne retenant que deux concepts fondamentaux rencontrés le plus souvent dans les discours et les écrits : la francophonie et la Francophonie. Une même acception interpellant pourtant ici deux terminologies se différenciant orthographiquement. En effet, la francophonie avec un $f$ minuscule renvoie à l'ensemble des personnes qui utilisent la langue française. Alors que la Francophonie avec un $f$ majuscule réfère à l'ensemble des institutions intergouvernementales ou gouvernementales dont le rôle principal est de promouvoir la langue française et de faire connaître la culture francophone sur une échelle mondiale.

L'extension de sens du mot francophonie est liée à un sentiment de la langue qui tend à la fois à maintenir un équilibre entre la particularisation des différences et la domination d'une variété de référence. Les ouvrages portant sur l'histoire de la francophonie privilégieront un regard à la fois institutionnel et géographique et de ce fait confirmeront une vision géopolitique de la francophonie.

Même à l'intérieur de l'hexagone, il existe entre les régions administratives de la France métropolitaine et les régions linguistiques un conflit sur le plan des représentations mentales analogue à celui de la Francophonie institutionnelle et la pratique généralisée de la langue française comme langue vernaculaire.

\section{Le régionalisme linguistique}

Nous observons un décalage dans l'usage de français régional et celui de langue régionale ${ }^{4}$. Dans le premier cas, on considère une variété et dans l'autre un usage standardisé. De la

${ }^{4}$ La locution langue régionale a suscité un débat important il y a quelques années. Il serait pertinent de se demander s'il faut employer en même temps langue régionale et français régional. En se reportant à la Loi Deixone 
même manière, en français d'Afrique, la sous-région représente "un ensemble géographique homogène comprenant plusieurs Etats" (Frey 167). Ce français diversifié qui se développe "n'est plus la langue de l'élite, ni celle du pouvoir, ni celle de la sacro-sainte promotion sociale; c'est devenu l'idiome véhiculaire d'un grand nombre de locuteurs africains" (Dumont 33). Le français par définition pluriel dans ses emplois finit par devenir multiple dans ses pratiques. Pour reprendre Dumont, nous pouvons "parler de français polymorphe, d'une "francopolyphonie"'" (Dumont 33). Ainsi, la francophonie africaine tire toute sa richesse et toute son originalité non seulement à l'extérieur de la langue, mais aussi dans le rapport qu'elle entretient avec les autres langues en contact avec le français. Si la notion de français régional connaît un tel engouement c'est parce que les variétés géographiques du français circulent davantage faisant découvrir des réalités linguistiques à l'extérieur d'une localité. "A partir de 1969, c'est l'expression langue régionale qui unifie le discours législatif, sans que les expressions local, locaux disparaissent cependant, comme dans les langues et dialectes locaux en 1974 et 1971, ou bien cultures locales ou régionales en 1975 et 1976" (Tabouret-Keller 104). La reconfiguration de la francophonie, et ses conséquences sur le français général, la loi de la décentralisation en France, la politique européenne, qui conduit au renforcement des pouvoirs supranationaux, impliquent de s'interroger sur la définition d'un régionalisme issu de la Révolution et de l'Empire (Le Dû 30). Le régionalisme francophone est donc passé d'une vision provinciale à une vision internationale par l'essor de la Francophonie institutionnelle qui a conduit à la reconnaissance des variétés nationales du français. Il semble intéressant de revenir de manière exhaustive sur la notion de langue pour mieux appréhender cet héritage colonial et ce contexte socioculturel de domination. Pour reprendre les propos de Bourdieu "parler de la langue, c'est accepter tacitement la définition de la langue officielle d'une unité politique" (Bourdieu 27). Cette conception de la langue permet d'asseoir un pouvoir, elle prétend ainsi niveler les différences qui existent entre les locuteurs de la langue. Par exemple en Afrique noire ou à Madagascar, depuis le temps de l'administration française et de la littérature coloniale, la langue française s'est tenue du côté du pouvoir. Mais cette représentation de la langue a considérablement évolué puisque son histoire n'est pas partout identique ; ainsi au Québec on lutte actuellement pour une autonomisation de la langue.

Les diverses théories sur la/les langues ont fait émerger d'autres notions ; parmi lesquelles celle de "surconscience linguistique" proposée par Lise Gauvin, "c'est-à-dire une conscience particulière de la langue qui devient ainsi un lieu de réflexion privilégié et un désir d'interroger la nature du langage et de dépasser le simple discours ethnographique. Cette surconscience est aussi une conscience de la langue comme espace de fiction voire de friction : soit un imaginaire de et par la langue" (Gauvin 19). Considérant que le "dénominateur

sur "L'enseignement des langues et dialectes locaux" (n`51-46 du 11 janvier 1951) nous constatons que le terme unificateur est local, à titre d'exemple nous pouvons citer : langues ethniques de France, langues de France, langues identitaires, langues et dialectes à extension régionale. 
commun des littératures dites émergentes, et notamment des littératures francophones, est de proposer, au cœur de leur problématique identitaire, une réflexion sur la langue et sur la manière dont s'articulent les rapports langues/littératures dans ces contextes différents", considérant que l'écrivain francophone est contraint de "penser sa langue", elle propose de nommer ces littératures "littératures de l'intranquillité", car elles se mettent à distance de la langue française.

Par ailleurs, il appartient à Jean-Marie Klinkenberg (71-80) d'avoir développé la notion $\mathrm{d}^{\prime \prime}$ "insécurité linguistique" des collectivités francophones périphériques. Il montre que face au centre constitué par Paris, les écrivains des zones francophones dominées adoptent deux directions opposées : soit une stratégie centripète (vers l'autonomisation, et la création d'un champ culturel distinct), soit une stratégie centrifuge ("un effort d'assimilation au champ parisien ou au moins le désir de reconnaissance de la part des instances de consécration de ce centre") (Klinkenberg 52). Ces théories ont en commun d'interroger la question de la langue écriture. Ce qui apparaissait auparavant comme l'élément rassembleur, le point d'arrivée : écrire en français, devient le point de départ de nouvelles approches littéraires.

Ces deux perspectives ont en apparence une orientation contradictoire, puisque l'une est centrifuge, cherchant à décentrer les études littéraires francophones, et l'autre est centripète, proposant de relier, de mettre en relation les langues, c'est-à-dire d'explorer les zones de contacts; mais ces deux axes de réflexion explorent en réalité le même chemin, celui du pluralisme.

\section{Une dimension utopique dans la Francophonie}

\section{Définition de l'utopie}

Cette clarification s'avère indispensable en raison du sens prêté par le langage courant à ce concept. Dans une acception péjorative, Utopie est assimilée à un projet irréalisable, une chimère, une rêverie. Le concept a été inventé par Thomas More ${ }^{5}$ au début du XVIème siècle qui présentait son modèle du "meilleur gouvernement possible" dans une Angleterre traversée par des crises sociales répétées et le mal-vivre. Le mot "Utopie", vient du grec "ou" qui signifie non et de «topos", qui signifie lieu, ce qui nous donnerait "Utopie = le lieu qui n'existe pas" (Petit Larousse 2002). Ce sens étymologique diffère du sens philosophique,

\footnotetext{
${ }^{5}$ Le terme d'utopia est un néologisme grec inventé par Thomas More en 1516 pour désigner la société idéale qu'il décrit dans son œuvre (en latin) Utopia. Il est traduit en français par utopie. Ce terme se compose de la préposition négative grecque ou et du mot topos qui signifie lieu. Le sens d'Utopie est "sans lieu », "qui ne se trouve nulle part». L'œuvre de More a pour caractéristique d'être, d'une part, un récit de voyage et la description d'un lieu fictif (utopia) et, d'autre part, un projet d'établissement rationnel d'une société idéale (Eutopia). Ces aspects du texte de l'auteur ont amené à qualifier d'utopie des œuvres très différentes.
} 
donné par le Petit Larousse : société idéale, mais imaginaire, telle que la conçoit et la décrit un auteur donné. Les utopies écrit le même document : "décrivent le fonctionnement de sociétés parfaites, dont on suppose l'existence en un lieu généralement clos (une cité, une île). Fournissant des arguments pour la critique de l'ordre existant, elles peuvent aussi s'offrir comme des modèles pour l'établissement de communautés heureuses". Les auteurs d'utopie sont nombreux : Platon, T Bacon, Campanella, T More, Morelly, Saint-Simon, Fournier, etc. Un autre sens étymologique est proposé par Verzy Szacki, chercheur polonais qui a consacré une grande partie de sa vie à l'étude de la problématique de l'utopie, surtout dans sa relation avec la tradition, c'est-à- dire, " ce qui est là déjà, et qui ne veut pas céder la place à ce qui arrive". Szacki n'a pas ici la même approche en ce qui concerne le préfixe "u". Selon ce dernier, "u" proviendrait du grec "ou" qui signifierait "non", ou encore de "eu", ce qui signifierait "bon". Ainsi " Utopie" serait polysémique. Ce mot pourrait signifier tantôt "le lieu qui n'existe pas", tantôt "le lieu confortable". Chaque fois qu'un homme se fixe un grand et noble objectif susceptible d'apporter des changements profonds et positifs à la société, il sera accusé d'utopiste. Dans ce cas alors, il faut accepter de l'être, puisque aucun progrès n'est concevable sans une dose d'utopie, c'est à dire sans le désir de vivre mieux demain qu'aujourd'hui. L'utopie, est la seule chose qui justifie l'expérience et nous le savons, la science aussi procède par expérience. L'utopie est un rêve qui devient système de pensées et fonde une doctrine, une idéologie capable de servir de guide à l'action humaine et sociale. C'est ce qui nous fait dire que toute utopie est "humaine" et c'est un mérite pour cet animal social qu'est l'homme d'avoir une telle capacité : celle de rêver et de travailler pour la matérialisation de son rêve. Comprendre l'utopie comme expérience, conduit nécessairement, à reconnaître sa proximité avec la science, qui est un processus d'observation, d'élaboration d'hypothèses, d'analyse, de théorisation et de vérification, et même si elle semble plus proche de la fantaisie (de fantasme). Comme en sciences, toute utopie, renferme en soit la volonté profonde de mieux connaître l'être humain afin de le rendre plus heureux. En d'autres termes, on pourrait avancer ce qui suit : donner à l'être humain la possibilité de vivre mieux, c'est lui permettre de choisir autre chose, autre situation que celle qu'il vit dans le présent. Offrir cette possibilité c'est proposer une alternative pour "demain", ce qui n'est possible que grâce à une utopie qui suppose la critique de l'ordre existant et la proposition d'un nouvel ordre, conçu et apprécié comme meilleur par rapport à celui existant.

\section{La Francophonie : une utopie?}

Cette idée francophone de l'unité (autour d'une langue et des valeurs) dans la diversité (des manières d'être) à travers le dialogue n'a pas connu le sort d'une utopie ni d'une uchronie ; elle s'est matérialisée institutionnellement par la vertu d'une force opérative capable de poser dans le monde des déterminations véritablement nouvelles, à savoir : l'action, qui, dans le cadre d'une vie collective où elle suppose la communication, le débat, l'adhésion, l'accord et l'adoption d'une réglementation ou la proclamation d'une loi, passe obligatoirement par la 
parole (Ladrière 385-390). La parole comme dimension de la force opérative qu'est l'action ne se réduit pas à l'intuition de l'idéal (ou du projet), elle se déploie aussi à travers l'argumentation idéologique au service des institutions agissantes pour transformer le réel. Le paradoxe de la francophonie signifie l'écart, mais aussi la dialectique (le va-et-vient) entre le (projet) francophone et la francophonie idéologique/institutionnelle active dans le monde et aux prises avec la force des choses. La francophonie idéologique/institutionnelle évolue positivement en s'efforçant d'inscrire dans le monde la philosophie (ou le projet) francophone. La littérature francophone à son tour se précise (en devenant philosophie des dimensions existentielles majeures: la langue, la culture, l'économie, la politique, l'écologie,...) au regard des imperfections concrètes de la francophonie active qu'elle cherche à éclairer conceptuellement. Mais cette dialectique ne doit nullement nous cacher l'écart, le négatif du paradoxe qui consiste en ceci que la francophonie active (a) fait parfois un usage mystificateur de l'idéel ou de l'idéal de manière à faire courir à ce dernier le risque de devenir sinon une utopie, du moins une idéologie (au sens négatif du terme). Les conclusions séparées, celle de Abdou Diouf et celle de Dominique Wolton à la fin du 40è numéros de la revue Hermès consacré au thème de "Francophonie et mondialisation" illustrent cette tension, ce distinguo. Il y a bel et bien une mise en perspective cosmopolitique de la francophonie dans le monde pour autant que la francophonie constitue un microcosme, un modèle réduit $\mathrm{du}$ monde, une organisation ouverte et représentative de tous les continents, des diverses religions, de plusieurs langues, et donc un laboratoire de la solidarité (cf. Abdou Diouf). Mais il y a aussi une timidité, une bureaucratie, un cléricalisme, un manque de militantisme... (Dominique Wolton) qui empêchent une émergence significative du projet francophone de déconstruction de la mondialisation unilatérale/utilitariste au profit de celle multilatérale/humaniste. Dominique Wolton nous rappelle à travers tous les chapitres de son livre ambitieux Demain la francophonie, qui se veut aussi un mode d'emploi, que la francophonie est surtout une utopie concrète qui transcende bien des frontières idéologiques, à condition d'en combattre les idées reçues et d'en faire un levier politique, par exemple pour la politique extérieure de la France...

\section{Senghor : concepteur et architecte de la Francophonie}

En tant que projet humain, la francophonie constitue une belle et grande utopie, dans le sens de Thomas More. Et seul un homme visionnaire, doublé d'un humanisme engageant et engagé, est capable de mettre toute sa force, toute son intelligence et toute sa vie à le concevoir et à le théoriser. La francophonie n'est pas une affaire de bons colonisés nostalgiques du passé. Elle n'est ni un projet revanchard et hégémonique d'une ancienne puissance colonisatrice. La Francophonie dans sa forme et sa compréhension actuelles, est l'œuvre du génie de cet homme, qui fut à la fois politique et penseur. Nous ne voulons pas insinuer qu'il fut le seul à vouloir de la Francophonie. Les Français y pensaient, le voulaient, 
mais pour des raisons faciles à deviner, ne voulaient pas regarder le problème en face, comme l'a fait remarquer en 1966, Jean de Broglie, dans un article paru dans Le Monde. Réticence et prudence. Jacques Chirac aussi le reconnaîtra en 1984 au Québec en ces termes : "La francophonie est le résultat de combats qui ont été menés la plupart du temps en dehors de la France. Cela nous donne à nous autres Français, une modestie". Du côté des hommes politiques africains, on suspectait un néo-impérialisme français. Un contexte peu favorable, pour ne pas dire hostile, à tout discours globalisant. Et pourtant c'est dans un tel contexte que Senghor choisit d'agir! "Il s'est clairement fait l'avocat et le philosophe de cette idée". La Francophonie est donc une alternative à l'indifférence réciproque, à l'isolement, au reniement du passé fait de violences et d'humiliation. Une alternative à l'émiettement de l'intelligence humaine. Et puisqu'elle est une alternative ayant pour fondement la volonté de changer un type de relation existante, relation que d'aucuns diront de dominés et de dominants, où il n'y aurait point de place pour le dialogue et les échanges humains. Senghor a certainement plus tôt que beaucoup d'intellectuels et de politiques de son temps, compris que l'Histoire ne peut être réécrite. Mais l'avenir se construit et se fait par les hommes. Et s'il faut agir, c'est sur l'avenir. Senghor a vécu la colonisation, a lutté pour l'émancipation des peuples noirs, mais a choisi de se tourner vers l'avenir. Non pas pour lui-même, mais pour les hommes et pour l'histoire. Car : "les individus historiques sont ceux qui ont voulu et accompli non une chose imaginée et présumée, mais une chose juste et nécessaire et qu'ils l'ont compris parce qu'ils ont reçu intérieurement la révélation de ce qui est nécessaire et appartient réellement aux possibilités du temps" (Senghor 1988). Senghor fut un de ces hommes historiques, et la Francophonie, une chose juste et nécessaire en raison des contextes historiques. Il n'a pas conçu la Francophonie et œuvré pour elle, pour des fins personnelles. Il l'a fait pour les peuples parlant français, et désireux de travailler ensemble pour une vie meilleure.

\section{Conclusion :}

Ainsi, les années 60 marqueront le passage progressif de la tendance centrifuge universalisante (la rencontre des cultures et des langues) à la tendance centripète particularisante (la quête de l'identité) (Frey 2003) qui tend à souligner la variation. André Reboullet, Léopold SédarSenghor et d'autres sont perçus comme les premiers acteurs qui ont su lier les champs littéraire, politique, sociolinguistique et le développement des actions institutionnelles au sein de la Francophonie. Le terme Francophonie semble de plus en plus se spécialiser comme en témoigne ses différents usages. Bourguiba dans un discours en 1968 emploiera Francophonie en le chargeant de sens institutionnel alors que Senghor dénote l'appartenance de la Francophonie à la culture française. Charles de Gaulle connotera le terme d'un certain néocolonialisme qui renvoie à l'idée d'expansion de la puissance de la France. 


\section{Bibliographie :}

- Barrat, Jacques. Géopolitique de la Francophonie. Un nouveau souffle?

La Documentation française : Paris, 2004.

- Batho, Jacques. "Le français, La Francophonie et les autres". Politique étrangère. $\mathrm{n}^{\circ} 1$, janvier-mars, 2001.

- Boulanger, Jean-Claude. "Francophonie, un terme, des sens". Langues et linguistiques. $\mathrm{n}^{\circ} 11,1985$.

- Boulanger, Jean-Claude. "A propos du concept de régionalisme". Lexique. n 3, 1985.

- Boulanger, Jean-Claude. Les français régionaux: observations sur les recherches actuelles. Montréal : Office de la langue française, 1980.

- Boutros-Ghali, Boutros. Emanciper la Francophonie. L'Harmattan : Paris, 2002.

- Charles-Brun, Jean. Le régionalisme, Mireille Meyer. Julian Wright : Paris, CTSH, 2004.

- Chaudenson, Robert. Vers une révolution francophone. L'Harmattan : Paris, 1989.

Chauprade, Aymeric. L'espace économique francophone : pour une francophonie intégrale. Ellipses: Paris, 1996.

- Deniau, Xavier. La Francophonie. Paris : PUF, 1983.

- Dumont, Pierre. La Francophonie autrement. Héritage Senghorien? Et si le Faire l'emportait sur le Dire... L'Harmattan, 2008.

- Dumont, Pierre. L'interculturel dans l'espace francophone. L'Harmattan, 2001.

- Frey, Claude. "Identités lexicales et variétés de français en France et hors de France: tendances centripètes et centrifuges des formes et des cultures".

- Pierre, Nobel, Variations linguistiques Koinè, dialectes, français régionaux. Besançon: Presses Universitaires de France-Comté, 2003.

- Gauvin, Lise. La fabrique de la langue. Paris : Seuil, 2003.

- Klinkenberg, Jean-Marie. "Autour du concept de langue majeure". Lise Gauvin et Jean-Paul Bertrand Littératures mineures en langue majeure, PUM, 2003.

- Latin, Danièle, Claude, Poirier and coll. Nathalie, Bacon, Jean, Bédard. Contacts de langues et identités culturelles, perspectives lexicographiques Actes des quatrièmes Journées scientifiques du réseau "Etude du français en francophonie". AUF et les Presses de l'Université Laval : Québec, 2000.

- Le Dû, Jean. "La langue bretonne aujourd'hui". Philippe Blanchet, Roland Breton et Harold Schiffman. Les langues régionales de France: un état des lieux à la veille du XXIe, actes d'un colloque organisé à "The University of Pennsylvania, Philadelphia, USA". Peeters, Louvain-laNeuve, 1999.

- More, Thomas. L'Utopie ou le Traité de la meilleure forme de gouvernement. Traduit par Marie, Delcourt. Garnier-Flammarion : Paris, 1987.

- More, Thomas. Utopie. Trad. V. Stouvenel and M. Bottigelli-Tisserand. Paris : 1976. 
- Pöll, Bernard. Francophonies périphériques, Histoire, statut et profil des principales variétés du français hors de France. L'Harmattan : Paris, 2001.

- Reclus, Onésime. France, Algérie et colonies. Hachette : Paris, 1886.

- Rezeau, Pierre. "L'influence sur le français de France du français venu d'ailleurs". Danièle, Latin, Claude, Poitier and coll. Nathalie, Bacon, Jean, Bédard, 2000.

- Senghor, Léopold Sédar. Ce que je crois : Négritude, francité et civilisation de l'universel. Grasset, 1988.

- Senghor, Léopold Sédar. Négritude et civilisation de l'universel, discours, conférences. Le Seuil, 1977.

- Tabouret-Keller, André. "L'existence incertaine des langues régionales en France". Philippe Blanchet, Roland Breton et Harold Schiffman. Les langues régionales de France : un état des lieux à la veille $d u$ XXIe, actes d'un colloque organisé à l'université de Pennsylvanie, Philadelphia, USA. Peeters, Louvain-la-Neuve, 1999.

- Tiesse, Anne-Marie. La création des identités nationales. Seuil : Paris, 2001.

- Wolton, Dominique. Demain la Francophonie. Flammarion : Paris, 2006. 